\title{
Harmonic transfer functions based controllers for linear time-periodic systems
}

Transactions of the Institute of Measurement and Control $1-14$

(C) The Author(s) 2018

Reprints and permissions: sagepub.co.uk/journalsPermissions.nav DOI: $10.1177 / 0142331218778748$ journals.sagepub.com/home/tim

@SAGE

\author{
Elvan Kuzucu Hidir', Ismail Uyanik ${ }^{2}$ (D) and Ömer Morgül ${ }^{3}$
}

\begin{abstract}
The analysis, identification and control of periodic systems has gained increasing interest during the last few decades due to the increased use of dynamical systems that exhibit periodic motion. The vast majority of these studies focus on the analysis and control problem for a known state-space formulation of the linear time-periodic (LTP) system. On the other hand, there are also some studies that focus on data-driven identification of LTP systems with unknown state-space formulations. However, most of these methods provide numerical estimates for the harmonic transfer functions (HTFs) of an LTP system that are extremely difficult to work with during controller design. The goal of this paper is to provide a simple controller design methodology for unknown LTP systems by utilizing so-called HTFs estimates. To this end, we first build a mathematical basis of LTP controller design for known LTP systems using the Nyquist diagrams and analytically derived HTFs. We propose a new methodology to design P-, PD- and PIDtype controllers for LTP systems using Nyquist diagrams and the eigenlocus of the HTFs. Having established the HTF-based controller design procedure, we extend our methodology to unknown LTP systems by presenting a new sum-of-cosine signal-based data-driven system identification method. We show that the proposed data-driven controller design method allows estimation of the HTFs and it provides simple tools for optimizing certain time-domain performance metrics. We provide numerical examples for both known and unknown LTP system cases to illustrate the performance of the proposed controller design methodology.
\end{abstract}

\section{Keywords}

linear time-periodic systems, harmonic transfer functions, Nyquist stability criterion, controller design

\section{Introduction}

The increased interest in periodic motion has also increased the necessity to develop novel tools for the analysis, identification and control of periodic systems (Farkas, 2013; Sandberg et al., 2005). Some examples of such periodic systems are wind turbines (Allen et al., 2011; Bottasso and Cacciola, 2015), helicopter rotors (Hwang, 1997; Siddiqi, 2001), power systems (Kwon et al., 2017; Mollerstedt and Bernhardsson, 2000a) and some nonlinear systems that exhibit periodic behavior around a stable limit cycle (Logan et al., 2016; Sracic and Allen, 2011; Uyanik, 2017). Note that standard linear timeinvariant (LTI) analysis tools cannot capture the periodic nature of the system dynamics for these examples. For instance, the switching nature in power systems may result in harmonic responses that should be considered carefully using periodic system analysis methods to guarantee the stability of such systems, see Möllerstedt (2000).

One of the most important tools on the analysis of periodic systems was proposed by Wereley (1990), which models the input-output relationship of linear time-periodic (LTP) systems using a linear operator called harmonic transfer functions (HTFs). HTFs are analogous to the input-output transfer functions of LTI systems (Bittanti and Colaneri, 2000). Different than a classical LTI transfer function, HTFs are composed of multiple modulated LTI transfer functions corresponding to each harmonic response of an LTP system. Fortunately, identification of HTFs for LTP systems received considerable attention from the scientific community and there are some available methods for the estimation of HTFs using input-output data of an LTP system (H1dır, 2017; Hwang, 1997; Siddiqi, 2001; Uyanik et al., 2016a). These methods mostly use chirp type (or single-sinusoid) input signals for system identification. On the other hand, there are also some methods that consider estimating a direct statespace model for LTP systems using subspace identification techniques (Goos and Pintelon, 2016; Uyanik et al., 2017, 2016b; Verhaegen and $\mathrm{Yu}, 1995)$.

The stability analysis of LTP systems is also not as mature as in the case of LTI systems. However, Floquet theory has

\footnotetext{
'Department of Electronics and Communication Engineering, Istanbul Technical University, Istanbul, Turkey

${ }^{2}$ Laboratory of Computational Sensing and Robotics, Johns Hopkins University, Baltimore, MD, USA

${ }^{3}$ Department of Electrical and Electronics Engineering, Bilkent University, Ankara, Turkey
}

\section{Corresponding author:}

Elvan Kuzucu Hidir, Department of Electronics and Communication Engineering, Istanbul Technical University, 34469 Istanbul, Turkey. Email: ehidir@itu.edu.tr 
contributed a lot to the stability analysis of periodic systems by proposing a change of coordinate transformation, where one can obtain a time-invariant state matrix (Richards, 1983). We note that in a more abstract setting the equivalence of an LTP system to an LTI system through Floquet transformation is valid under certain assumptions on the time scale, which trivially holds for continuous and discrete time systems. For more information on this subject, see e.g. DaCunha and Davis (2011) and the references therein. In addition, stability analysis can also be achieved using the Lyapunov theory (Owens et al., 2004) or Hill determinant methodology (Richards, 1983). However, one common disadvantage of these techniques is that they do not address the closed-loop stability problem of LTP systems. In the case of LTI systems, the Nyquist stability criterion is a very powerful technique to investigate closed-loop stability based on the eigenlocus of the system for a family of closed-loop gain parameters. Hall and Wereley (1990) showed that a similar analysis can be applied to LTP systems when they are lifted to an equivalent LTI system by using the principle of harmonic balance. However, this has only been shown for systems when the state-space matrices are available. Hence, it still remains unclear whether a similar analysis would hold for unknown LTP systems, whose HTFs can be estimated using data-driven system identification techniques.

The control problem of periodic systems has also received consideration from the control theory community (Bittanti et al., 1973). One of the most successful control strategy regarding the LTP systems is the use of linear quadratic regulator (LQR) controllers, which can provide periodic feedback gain to guarantee stability and robustness (Anderson and Moore, 2007; Athans, 1971). LQR controllers are especially favorable for the control of active vibrations in LTP systems such as wind turbines, helicopter robots and turbo machinery (Arcara et al., 1997; Jakobsen et al., 2013). However, one main assumption behind the use of LQR controllers is the availability of a state-space representation by using which one can develop state observers. Similar ideas have been developed by using static feedback gain controllers via Nyquist plots (Wereley, 1990) and some other power network system controllers (Bellinaso et al., 2011; Mollerstedt and Bernhardsson, 2000b; Scapini et al., 2012) when the statespace representation of the LTP system is given a priori. Despite the availability of some state-space identification methods for LTP systems (Uyanik et al., 2017; Verhaegen and $\mathrm{Yu}, 1995)$, the control problem based on estimated statespace models is still an open issue to the best of authors' knowledge. On the other hand, it is also very challenging to design controllers based on estimated HTFs, because an LTP system can include possibly infinite number of HTFs to accurately represent its input-output characteristics.

In this paper, we aim to perform stability analysis and controller design for LTP systems with known and unknown state-space formulations. As mentioned above, stability of a known LTP system can be simply checked by utilizing Floquet theory to examine the eigenvalues of the fundamental monodromy matrix. Similarly, one can use Lyapunov theory to investigate the stability of a known LTP system. However, both of these methods are highly challenging and it is even impossible to find an analytic representation of the state transition matrix in certain cases. One disadvantage of these methods is that they need to be repeated for each value of a feedback gain. In contrast to these techniques, Nyquist diagrams can provide a better analysis of the stability by using the eigenlocus of HTFs to examine the stability for a range of feedback gain values. Hence, we will first focus on how these techniques can be utilized for LTP systems in terms of HTFs to evaluate the closed-loop stability of periodic systems.

The closed-loop stability analysis of an LTP system with respect to certain feedback controllers becomes more challenging when the state-space formulation of the system is unknown. A second contribution of this work is to provide a data-driven system identification method to estimate HTFs of an unknown open-loop stable LTP system. Here, our goal is to construct the necessary HTF matrices to utilize the Nyquist stability criterion for the closed-loop system for a family of feedback controllers. To this end, we propose a sum-ofcosines input design-based system identification strategy, where we estimate the HTFs of the unknown LTP system using the fundamental principles from the frequency response functions of LTP systems. The proposed method provides considerable advantages when compared with single-cosinebased (Hwang, 1997) and chirp-input-based (Siddiqi, 2001; Uyanik et al., 2015) methods in terms of number of tests required and the estimation accuracy, respectively.

Once we have an accurate evaluation of the stability, we can design controllers both to ensure stability as well as to increase system performance of both the known and unknown LTP systems. Note that most of the available controller design methods for LTP systems exclusively focus on LQR controllers (Arcara et al., 1997; Jakobsen et al., 2013; Mckillip, 1985). There are two main concerns for using LQRtype controllers in this study. First, we want to focus on unknown LTP systems, where the state-space formulation is not available. Second, we seek to increase some time-domain system performance metrics such as the gain margin (GM) and phase margin (PM), which might not be possible to achieve by using only a feedback gain-type controller.

To summarize, our main contributions in this paper are as follows. First, we analyze the stability of known LTP systems by utilizing the Nyquist stability criterion based on HTFs. Then, we design different type of controllers, such as P, PD and PID, to stabilize the LTP system and enhance its performance. To investigate the stability for a range of feedback gain, Nyquist diagrams provide better analysis via eigenlocus of HTFs. The key contribution at this point is that we separate the $K_{p}$ parameter of a controller (such as the PD case) as a feedback gain and evaluate the stability for a range of $K_{p}$ parameter in a single Nyquist stability test. Hence, if we seek to optimize the system performance for a PD-type controller, which normally requires a two-dimensional optimization problem due to unknown $K_{p}$ and $K_{d}$, we only need to perform a one-dimensional optimization, because the Nyquist test provides the stability results with respect to $K_{p}$. The second contribution of this paper is the controller design procedure for unknown LTP systems. To this end, we first present a new sum-of-cosines-based system identification method for LTP system, which drastically reduces the number of required experiments. We also provide a systematic controller design methodology to utilize these estimated HTFs for designing 
controllers for the unknown LTP system. We also provide a method for optimizing system performance such as the GM, PM and settling time by optimizing control parameters for the unknown LTP system.

This paper is outlined as follows. We first formulate our problem definition including some preliminary background introduction regarding the LTP systems. Then, we explain the concept of HTFs including the analytical derivation when the state-space structure is known and the data-driven estimation for an unknown stable LTP system. Then, we detail how to investigate the stability of a known LTP system using the Nyquist criterion. We also explain how to design controllers based on the HTFs for known and unknown LTP systems. We provide numerical examples to illustrate the applicability of our methodology for both known and unknown LTP system examples. Finally, we provide some concluding remarks.

\section{Problem definition}

This paper focuses on the analysis and control of LTP systems, most of which can be represented in the form

$$
\begin{aligned}
& \dot{x}(t)=A(t) x(t)+B(t) u(t) \\
& y(t)=C(t) x(t)+D(t) u(t)
\end{aligned}
$$

where $\quad A(t) \in \mathbb{R}^{n \times n}, \quad B(t) \in \mathbb{R}^{n \times m}, \quad C(t) \in \mathbb{R}^{p \times n} \quad$ and $D(t) \in \mathbb{R}^{p \times m}$ are all $T$ periodic, i.e. $A(t+N T)=A(t)$ for any integer value of $N$. Here, $T>0$ is called the fundamental period of the system, which is the minimum common period for all state matrices of (1). Similarly, the fundamental frequency (pumping frequency) of the LTP system can be defined as $\omega_{0}=2 \pi / T$. In addition, $x(t) \in \mathbb{R}^{n}$ represents the state vector, $u(t) \in \mathbb{R}^{m}$ represents the input and $y(t) \in \mathbb{R}^{p}$ represents the output.

Considering the definition of the LTP system that we described above, this paper aims to attack two fundamental problems associated with the stability analysis and control of these systems.

Problem 1: Given the state-space system matrices $A(t), B(t)$, $C(t)$ and $D(t)$ for an LTP system of the form (1), (how) can we design controllers for such LTP systems both to ensure stability and to increase system performance by utilizing concepts from the Nyquist stability criterion?

As a remedy to this problem, in the first part of the paper, we focus on lifting an LTP system to an equivalent (higherdimensional) LTI system using the principle of harmonic balance. The key idea here originates from the frequency response characteristics of the LTP systems. When a complex exponential input signal is applied to an LTP system, the output includes complex exponentials both in the input frequency as well as in the harmonics of the periodic (pumping) frequency of the system. Wereley (1990) developed the concept of HTFs to represent each of these harmonic responses with a distinct transfer function. From this perspective, the HTFs allow the representation of an LTP system as the superposition of multiple modulated LTI systems. Based on this fact, we use HTFs as a frequency domain lifting method for LTP systems to find LTI equivalents, such that we can utilize LTI stability analysis and control techniques for the LTP systems as well.

Problem 2: Given an open-loop stable LTP system with unknown state-space matrices, (how) can we design performance increasing controllers ensuring that the closed-loop system is also stable?

As a solution to this problem, we propose a data-driven system identification approach to first estimate the HTFs of the unknown LTP system and then apply the similar stability analysis and control procedure from the solution of Problem 1. One challenge here is that the available LTI system identification methods in the literature cannot be used directly for the estimation of HTFs for an LTP system. Hence, we utilize a new data-driven system identification method based on sum-of-cosines input design methodology to estimate the HTFs of an LTP system using frequency response data. Once we have the estimates for the HTFs, the rest of the problem will be equivalent to intermediate steps of Problem 1, because we will use estimated HTFs instead of those that are derived using state-space formulation.

\section{HTFs}

The concept of HTFs was first developed by Wereley (1990) to represent the frequency response characteristics of LTP systems. Wereley (1990) defined the concept of exponentially modulated periodic (EMP) signals as a signal space for the input and output data of the LTP systems. This section first shows how one can derive the HTFs of an LTP systems given the time-periodic state-space formulation. This will also serve as a frequency-domain lifting method by using which we can obtain higher-dimensional LTI equivalents for the original LTP system. Then, the second part of this section proposes a data-driven system identification method for the estimation of HTFs without having a priori information about the statespace formulation of the original unknown LTP system.

\section{Derivation of HTFs using the known state-space formulation}

The objective of this section is to present the basic derivations for HTFs (see Wereley and Hall, 1990). To start with, we first give a mathematical representation of the EMP signals as

$$
u(t)=\sum_{n \in \mathbb{Z}} u_{n} e^{s_{n} t}
$$

as a common signal space for input-output data of the LTP systems, where $s_{n}=s+j n \omega_{0}, \forall n \in \mathbb{Z}$ and $s \in \mathbb{C}$. Different than regular complex exponential signals, EMP signals include another exponential term that creates the modulation of input signals with the integer multiples of pumping frequency of the system.

One fundamental property of using EMP signals for LTP systems is that both the state and output vectors can be represented as EMP signals at steady state (Wereley, 1990). Hence, we can write the state vector (and, hence, its derivative) as given below 


$$
x(t)=\sum_{n=-\infty}^{\infty} x_{n} e^{s_{n} t}, \quad \dot{x}(t)=\sum_{n=-\infty}^{\infty} s_{n} x_{n} e^{s_{n} t}
$$

Similarly, the output signal, $y(t)$ can also be represented as

$$
y(t)=\sum_{n=-\infty}^{\infty} y_{n} e^{s_{n} t}
$$

Different than input, output and state vectors, the system matrices are regular time-periodic signal matrices and, hence, they can be simply represented using complex Fourier series expansions as

$$
\begin{aligned}
& A(t)=\sum_{n=-\infty}^{\infty} A_{n} e^{j n \omega_{0} t}, \quad B(t)=\sum_{n=-\infty}^{\infty} B_{n} e^{j n \omega_{0} t} \\
& C(t)=\sum_{n=-\infty}^{\infty} C_{n} e^{j n \omega_{0} t}, \quad D(t)=\sum_{n=-\infty}^{\infty} D_{n} e^{j n \omega_{0} t}
\end{aligned}
$$

The derivations of HTFs start by plugging in these expansions into the state-space LTP system form given in (1). We start by expanding the state evaluation equation of (1) as

$$
\sum_{n=-\infty}^{\infty} s_{n} x_{n} e^{s_{n} t}=\sum_{n, m=-\infty}^{\infty} A_{n-m} x_{m} e^{s_{n} t}+\sum_{n, m=-\infty}^{\infty} B_{n-m} u_{m} e^{s_{n} t}
$$

Similarly, plugging in the expansions into the output equation of (1) yields the equation

$$
\sum_{n=-\infty}^{\infty} y_{n} e^{s_{n} t}=\sum_{n, m=-\infty}^{\infty} C_{n-m} x_{m} e^{s_{n} t}+\sum_{n, m=-\infty}^{\infty} D_{n-m} u_{m} e^{s_{n} t}
$$

Simplification and reorganization of the terms in (7) and (8) yields

$$
\begin{aligned}
& 0=\sum_{n=-\infty}^{\infty}\left\{s_{n} x_{n}-\sum_{m=-\infty}^{\infty} A_{n-m} x_{m}-\sum_{m=-\infty}^{\infty} B_{n-m} u_{m}\right\} e^{s_{n} t} \\
& 0=\sum_{n=-\infty}^{\infty}\left\{y_{n}-\sum_{m=-\infty}^{\infty} C_{n-m} x_{m}-\sum_{m=-\infty}^{\infty} D_{n-m} u_{m}\right\} e^{s_{n} t}
\end{aligned}
$$

One of the very special properties of (9) is that the exponential terms outside the brackets constitute basis vectors in $L_{2}[0, T]$ space. By using this property on (9), we obtain the following:

$$
\begin{gathered}
s_{n} x_{n}=\sum_{m=-\infty}^{\infty} A_{n-m} x_{m}-\sum_{m=-\infty}^{\infty} B_{n-m} u_{m} \\
y_{n}=\sum_{m=-\infty}^{\infty} C_{n-m} x_{m}-\sum_{m=-\infty}^{\infty} D_{n-m} u_{m}
\end{gathered}
$$

$\forall n \in Z$ and it is called the principle of harmonic balance.

Note that (10) defines infinitely many equations for the representation of frequency response characteristics of LTP systems. For the sake of obtaining a more intuitional representation, these equations can be combined in a single equation using doubly infinite (semi-)Toeplitz matrices. To accomplish this, we first define the doubly infinite input, output and state vectors as

$$
\begin{aligned}
& \mathcal{U}(s)=\left[\ldots, u_{-l}^{\mathrm{T}}(s), u_{0}^{\mathrm{T}}(s), u_{l}^{\mathrm{T}}(s), \ldots\right]^{\mathrm{T}} \\
& \mathcal{Y}(s)=\left[\ldots, y_{-l}^{\mathrm{T}}(s), y_{0}^{\mathrm{T}}(s), y_{l}^{\mathrm{T}}(s), \ldots\right]^{\mathrm{T}} \\
& \mathcal{X}(s)=\left[\ldots, x_{-l}^{\mathrm{T}}(s), x_{0}^{\mathrm{T}}(s), x_{l}^{\mathrm{T}}(s), \ldots\right]^{\mathrm{T}}
\end{aligned}
$$

where $u_{n}(s), y_{n}(s)$ and $x_{n}(s)$ correspond to the $n$th harmonic component of $u(t), y(t)$ and $x(t)$, respectively, and the superscript $\mathrm{T}$ denotes the transpose. Using these new definitions for input, output and state signals, the system of equations in (10) can be written as a single doubly infinite matrix equation as

$$
\begin{gathered}
s \mathcal{X}=(\mathcal{A}-\mathcal{N}) \mathcal{X}+\mathcal{B U} \\
\mathcal{Y}=\mathcal{C X}+\mathcal{D} \mathcal{U}
\end{gathered}
$$

Here the $T$-periodic state matrix, $A(t)$, is represented as a doubly infinite (semi-)Toeplitz matrix in terms of its complex Fourier series coefficients as

$$
\mathcal{A}=\left[\begin{array}{cccccc}
\ddots & \vdots & \vdots & \vdots & \vdots & \\
\ldots & A_{0} & A_{-1} & A_{-2} & A_{-3} & \ldots \\
\ldots & A_{1} & A_{0} & A_{-1} & A_{-2} & \ldots \\
\ldots & A_{2} & A_{1} & A_{0} & A_{-1} & \ldots \\
\ldots & A_{3} & A_{2} & A_{1} & A_{0} & \ldots \\
& \vdots & \vdots & \vdots & \vdots & \ddots
\end{array}\right]
$$

Similarly, $\mathcal{B}, \mathcal{C}$ and $\mathcal{D}$ are obtained using the complex Fourier series coefficients of $B(t), C(t)$ and $D(t)$, respectively. In addition to these, another doubly infinite (semi-)Toeplitz matrix $\mathcal{N}$ is constructed to include the frequency modulation terms regarding to EMP signals as

$$
\mathcal{N}=\left[\begin{array}{ccccc}
\ddots & \vdots & \vdots & \vdots & \\
\cdots & -j \omega_{0} I & 0 & 0 & \cdots \\
\cdots & 0 & 0 & 0 & \cdots \\
\cdots & 0 & 0 & j \omega_{0} I & \cdots \\
& \vdots & \vdots & \vdots & \ddots
\end{array}\right]
$$

where $I$ is the identity matrix with dimension of the state matrix $A(t)$.

One last step remaining in our analysis is to find a direct relation between the input and output signals using (14). This relationship is called as the HTFs, $\mathcal{H}(s)$, of the underlying LTP system and can be defined as

$$
\mathcal{Y}(s)=\mathcal{H}(s) \mathcal{U}(s)
$$

where

$$
\mathcal{H}(s)=\mathcal{C}[s I-(\mathcal{A}-\mathcal{N})]^{-1} \mathcal{B}+\mathcal{D}
$$

One important thing here is to note that the HTFs are also in a doubly infinite matrix form as 


$$
\mathcal{H}(s)=\left[\begin{array}{ccccc}
\ddots & \vdots & \vdots & \vdots & \\
\ldots & H_{0}\left(s-j \omega_{0}\right) & H_{-1}(s) & H_{-2}\left(s+j \omega_{0}\right) & \ldots \\
\ldots & H_{1}\left(s-j \omega_{0}\right) & H_{0}(s) & H_{-1}\left(s+j \omega_{0}\right) & \ldots \\
\ldots & H_{2}\left(s-j \omega_{0}\right) & H_{1}(s) & H_{0}\left(s+j \omega_{0}\right) & \ldots \\
& \vdots & \vdots & \vdots & \ddots
\end{array}\right]
$$

Here, the transfer function $H_{0}(s)$ defines the relationship between the input in the fundamental frequency to the output in the same frequency. Similarly, the other transfer functions define the relationship between different harmonic inputoutput pairs. One problem with the use of HTFs is their doubly infinite matrix structure. To overcome this issue, we truncate the HTFs after a certain number of harmonics for numerical computations.

\section{Data-driven identification of HTFs}

The previous section shows how the HTFs for an LTP system can be derived when the state-space matrices of the system are available. Different than the previous section, this section aims to estimate the HTFs using input-output data without having a priori knowledge of the state-space system matrices. In this section, we propose a data-driven system identification method for the estimation of HTFs using input-output data of an LTP system.

System identification with single-cosine excitations. In this section, we explain how HTFs can be estimated using the fundamental principles of LTP frequency response characteristics. Let the pumping frequency of an LTP system be $\omega_{0}$. Given an input signal $u(t)=\cos \left(\omega_{f} t\right)$, the LTP system generates sinusoidal signals both at the input frequency, $\omega_{f}$, as well as at the harmonics of the pumping frequency, $\omega_{0}$. Truncating the number of harmonics observed in the output, the inputoutput relationship of an LTP system can be written as

$$
\begin{gathered}
u(t)=\cos \left(\omega_{f} t\right) \\
y(t)=\sum_{n=-M}^{M} A_{n} \cos \left(\left(\omega_{f}+n \omega_{0}\right) t+\phi_{n}\right)
\end{gathered}
$$

Here, $M$ is the number of HTFs (after truncation), $A_{n}$ and $\phi_{n}$ are the gain and phase changes corresponding to input at $\omega_{f}$ for the $n$th HTF.

Considering the fundamental principles of the frequency response characteristics of the LTP systems, one can estimate these HTFs by observing the gain and phase between the input-output data of the LTP systems. Hence, HTFs can be computed as

$$
\hat{H}_{\mp n}\left(\omega_{f}\right)=\frac{U^{*}\left(\omega_{f}\right) Y\left(\omega_{f} \mp n \omega_{0}\right)}{U^{*}\left(\omega_{f}\right) U\left(\omega_{f}\right)}
$$

where $U(\omega)$ and $Y(\omega)$ represent Fourier transforms of $u(t)$ and $y(t)$, respectively.
System identification with sum-of-cosine input signal. Although the identification via single-cosine experiments is very basic and accurate in the sense that it is based directly on the fundamental principles of LTP frequency response characteristics, it requires many experiments to identify HTFs over a wide range of frequency bands. As a remedy to this problem, some researchers use frequency sweeping functions (chirp signals) to trigger all desired frequency bands in a single experiment (Siddiqi, 2001). However, chirp inputs are not preferred for LTP system identification for the following reasons, which might contaminate estimated frequency response functions.

- When a cosine input at a specific frequency, $\omega_{f}$, is given to an LTP system, it actually triggers both $\omega_{f}$ and $-\omega_{f}$. Hence, both of these frequencies will yield harmonics of the pumping frequency $\omega_{0}$ in the output. Therefore, the integer multiples of the pumping frequency should be avoided in the input, because the harmonics of the positive and negative sides will overlap and it will not be possible to discriminate between the effects of individual inputs without applying more specially designed input signals at the same frequency. One basic way to avoid these frequencies in a chirp signal is to divide the frequency band into sub-bands to exclude those frequencies; however, this will also increase the number of experiments required for system identification. In addition, it is not easy to guarantee that the designed chirp signal will avoid the specified frequency without using a high-order specially designed filters.

- Chirp signals yield frequency leakage during system identification, because they cover a continuous frequency range. Such frequency leakage problems decrease the accuracy of the estimations (Hwang, 1997).

- The system identification algorithms become much more complex than the single-cosine methods when chirp signals are used as the inputs.

As an alternative to chirp signals, we propose the use of sum-of-cosines signals, which also reduce the number of experiments required for system identification drastically and also eliminate the major disadvantages listed above for chirp signals. However, the classical sum-of-cosines strategy that is used for LTI systems cannot be applied directly to LTP systems, because one needs to consider the harmonics of an input signal while designing the sum-of-cosines input. The key idea here is that when we look at the frequency spectrum of the input-output signals after having the discrete Fourier transform, the spectrum is divided into small bins based on the sampling frequency and the signal length, which we call the frequency resolution, $f_{r}$. Hence, we first guarantee that input signal frequency is an integer multiple of $f_{r}$ to eliminate frequency leakage, which was one of the major disadvantages of using chirp signals. In addition, because we now have full control over the individual frequencies we can select, we exclude the frequency points that are integer multiples of the pumping frequency of the system. Hence, the frequency spectra will be divided into small frequency bands of the pumping 
Table I. Notation related to the sum-of-cosines input design

\begin{tabular}{lll}
\hline$f_{0}$ & $\triangleq$ & Fundamental frequency of LTP system. \\
$f_{r}$ & $\triangleq$ & Frequency resolution in frequency domain. \\
$f_{d r}$ & $\triangleq$ & Desired frequency resolution in input signal. \\
$f_{\max }$ & $\triangleq$ & Maximum value of frequency range of input signal. \\
$N_{s t}$ & $\triangleq$ & Number of frequencies contained in a test. \\
$N_{f b}$ & $\triangleq$ & Number of bands. \\
$N_{b t}$ & $\triangleq$ & Number of tests that can be performed in a band. \\
Definitions & $\frac{f_{0}}{2 f_{d r}}$ \\
$N_{s t}$ & $=$ & $\frac{f_{\max }}{f_{0}}$ \\
$N_{f b}$ & $=\frac{N_{s t}}{N_{f b}}$ \\
$N_{b t}$ & $=$
\end{tabular}

frequency. The key problem here is that once one chooses a frequency point on any frequency band, it actually triggers outputs in the same frequency point of all frequency bands owing to the harmonics that are multiples of the pumping frequency appearing in the output. Hence, we need to design a clever frequency scheduling strategy, where we can define a mathematical formulation for choosing the correct frequency values while designing our sum-of-cosines input signal. Before going into the details of this formulation, we first define some notation that will be used for our derivations in Table 1.

Based on the definitions given in Table 1, we first divide the desired frequency range for system identification into frequency bands of $f_{0}$. Hence, the number of frequency bands required for system identification can be found by dividing the maximum frequency range $f_{\max }$ to the pumping frequency of the system $f_{0}$. For the sake of clarity, the proposed frequency scheduling procedure is illustrated in Figure 1. In Figure 1, the sum-of-cosine input signal can only contain one of the frequencies that are of the same colour. The reason behind this selection procedure is to avoid any possible overlap between the harmonics of the input frequencies. In the case of such an overlap, the outputs of the corresponding input signals will coincide and basic empirical transfer function estimation methods would not work. Therefore, to avoid this issue, the input frequencies of these signals should not be in the same column that are illustrated with same colour in Figure 1. In addition, in Figure 1, there is a new red box in different column with the frequencies $f=0.21$. The first sliding frequency process is completed and the new sliding frequency for the input frequencies of sum-of-cosine input signals is started. This process is continued until the first half of the first band ends. Based on this principle, a sum-ofcosine input signal design formula is developed for the first and second parts of the frequency bands in

$$
\begin{aligned}
& u(t)=\sum_{l=1}^{N_{f b}} \sum_{k=0}^{N_{b t}-1} \cos \left(2 \pi\left(f_{r}+(l-1) \times\left(f_{p}+f_{d r}\right)+N_{f b} f_{d r} k\right) t\right) \\
& u(t)=\sum_{l=1}^{N_{f b}} \sum_{k=0}^{N_{b t}-1} \cos \left(2 \pi\left(f_{r}+(l-1) \times\left(f_{p}+f_{d r}\right)+N_{f b} f_{d r} k+\frac{f_{0}}{2}\right) t\right)
\end{aligned}
$$

\section{Stability analysis and control of known/ unknown LTP systems}

In this section, we aim to investigate the stability characteristics of LTP systems and to design stabilizing controllers that also increase system performance metrics. In standard LTI systems, the above goal can be achieved by a Nyquist stability test as a function of a feedback gain. Our goal here is to adapt this methodology for LTP systems, because LTP systems can be lifted to LTI equivalents, where they are represented with multiple modulated LTI systems. This idea necessitates the utilization of a Nyquist stability test in terms of HTFs of an LTP system. In addition to stability analysis, we also seek to

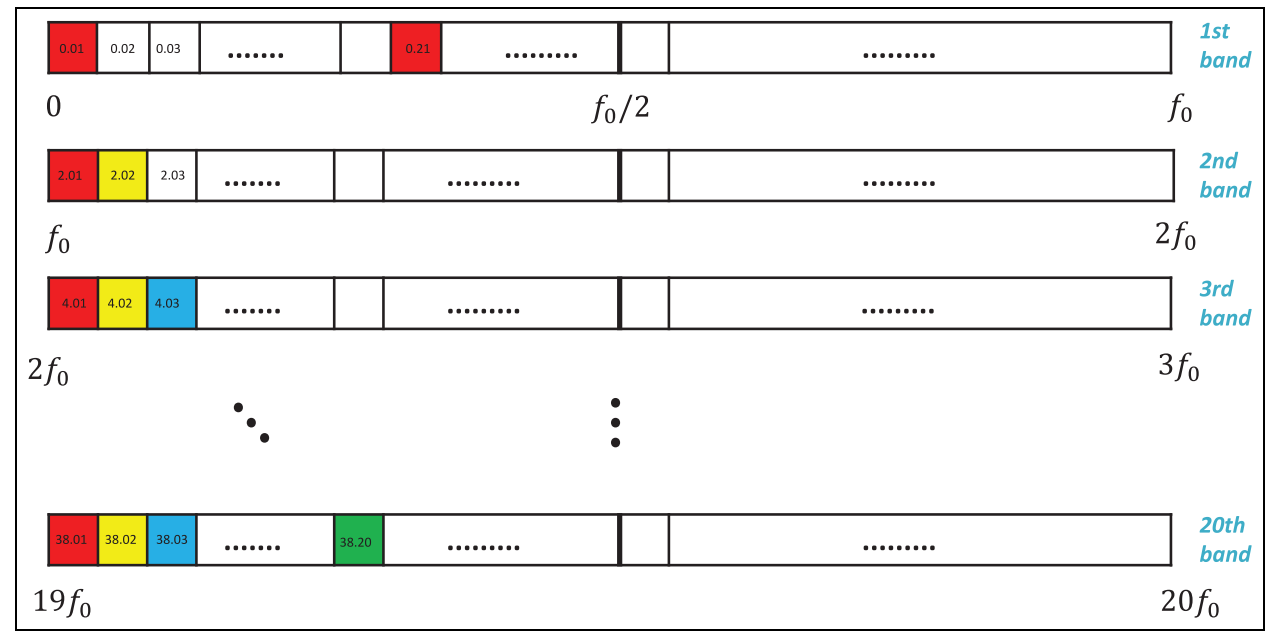

Figure I. Locations of the frequencies included in Sum of Cosine input signal. 
utilize the Nyquist plots of HTFs to design closed-loop controllers for the LTP system.

In this respect, we first show how one can construct the Nyquist plots for LTP systems via computing the eigenlocus of analytically derived HTFs. We then use these Nyquist plots to assess the stability characteristics of the known LTP system. One important point to note here is that Nyquist plots are obtained via the eigenlocus of the HTFs, which one can also estimate using data-driven techniques even without needing the state-space formulation of the original LTP system. To this end, we present how one can estimate the HTFs for an open-loop stable unknown LTP system and utilize these HTFs for the construction of Nyquist plots towards assessing the closed-loop stability of the overall system. After having a comprehensive understanding of the stability for both known/ unknown LTP systems, we show how one can design P-, PDand PID-type controllers to increase closed-loop system performance based on Nyquist diagrams.

\section{Stability analysis of known LTP Systems via analytically derived HTFs}

Stability analysis of LTP systems can be achieved via different methodologies such as Floquet theory (Richards, 1983), Lyapunov theory (Owens et al., 2004) or a Hill determinant technique. However, when there is a feedback gain, these techniques can only evaluate the stability of the closed-loop systems for only a specific value of gain. However, evaluating the stability of an LTP system for a range of feedback gains using a single stability test and choosing the optimal feedback gain that enhances system performance would make an important contribution to the controller design problem of LTP systems. Motivated by this, we focus on techniques such as Nyquist stability analysis that provide stability evaluation for a range of feedback gains. Note that for the system given in Figure 2, where $\mathcal{H}(s)$ represents an LTI transfer function with $p$ unstable poles, the closed-loop system is stable if and only if the Nyquist plot of $\mathcal{H}(s)$ encircles the point $-1 / k$ exactly $p$ times counterclockwise.

In the literature, the classical Nyquist stability criterion is first developed for single-input single-output (SISO) systems (Richards, 1983). Then, this notion is extended to the multiinput multi-output (MIMO) systems in various ways (Barmanj and Katzenelson, 1974; MacFarlane and Postlethwaite, 1977). In addition, MIMO Nyquist stability criterion which depends on eigenlocus of transfer function matrices is developed by (Desoer and Wang, 1980). Note that LTP systems can also be represented as MIMO LTI systems

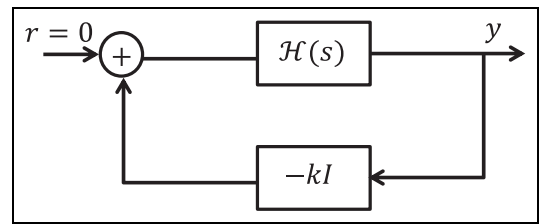

Figure 2. An illustration of the LTP system in closed loop with a feedback gain. when they are lifted with HTFs (Mollerstedt and Bernhardsson, 2000a). Hence, the Nyquist stability criterion for MIMO LTI systems can also be utilized to assess the stability of an LTP system. Thus, one can decide on the stability of the LTP system with respect to a range of feedback gains by using the Nyquist diagrams.

In this section, we explain how to apply the Nyquist stability criterion to evaluate the stability of a closed-loop LTP system illustrated in Figure 2. In an LTI system, closed-loop stability analysis with feedback gain $k$ can be examined by plotting a Nyquist contour of the transfer function $H_{0}(j \omega)$ for a frequency range $-\infty<\omega<\infty$ and counting encirclements of the point $-1 / k$. In principle, we can utilize the same idea for LTP systems, because they can always be represented with their MIMO LTI equivalents. However, owing to the possibly infinite number of harmonics that can be observed in an LTP system, the direct application of this idea might not be possible. Fortunately, this problem received considerable attention from the control theory community and we can adapt the Nyquist stability test to LTP systems by utilizing Theorem 1.

Theorem 1 (Hall and Wereley, 1990). Assume that there is a linear, periodic input output relation from $r$ to $y$ shown in Figure 2. Denote the eigenvalues of the doubly infinite Toeplitz for matrix $\mathcal{H}(s)$ given in (19) as $\left\{\lambda_{i}(s)\right\}_{i=1}^{\infty}$, for $s$ varying through the dotted contour in Figure 3 which is denoted as the fundamental strip $\overline{\mathcal{N}}_{f}$. These eigenvalues generate a number of closed curves in the complex plane that is called the eigenlocus of the HTFs. The feedback system illustrated in Figure 2 is stable from input, $r$, to the output, $y$, if and only if the total number of counterclockwise encirclements of the point $-1 / k$ of these closed curves is equal to the number of right half-plane poles of open loop $H T F, \mathcal{H}(s)$ in the fundamental strip.

In Wereley (1990), the eigenlocus of HTFs is parametrized by $s \in \bar{N}_{f}$ and Hill determinant is expressed as $|I+k \mathcal{H}(s)|$ regarding eigenvalues of HTFs as

$$
|I+k \mathcal{H}(s)|=\prod_{n=-\infty}^{\infty}\left(1+k \lambda_{n}(s)\right)
$$

Here, the product formulation is infinite owing to the doubly infinite transfer function matrix $\mathcal{H}(s)$ in (19). The number of harmonics here should be truncated for computational purposes, so we separate Equation (24) into the two parts as follows:

$$
|I+k \mathcal{H}(s)|=\prod_{n=-M}^{M}\left(1+k \lambda_{n}(s)\right) \prod_{n \notin[-M, M]}\left(1+k \lambda_{n}(s)\right)
$$

The first $M$ harmonics of the HTFs can be treated as significant parts and others should not be considered as necessary because they do not contribute to the encirclement of the point $-1 / k$. This is because the eigenvalues whose indices are greater than $M$ remain inside the unit disc, $D_{c}$. Thus, the number of eigenvalues of the first $M$ HTFs given in (25) can be counted as encirclements. As a result, the eigenlocus of truncated HTFs are obtained by computing eigenvalues of these HTFs through the Nyquist diagram, $\bar{N}_{f}$, illustrated in Figure 3 and plotted to the complex plane. The closed-loop stability of the system is provided if and only if the point 


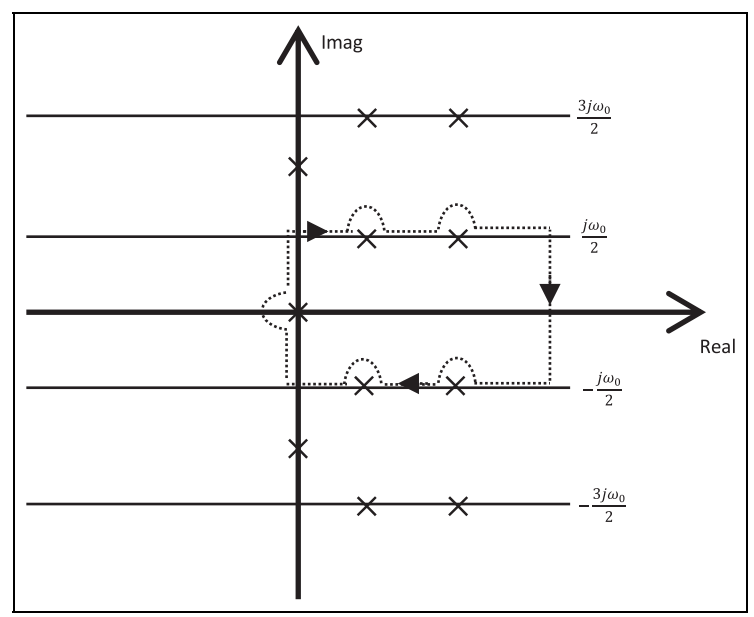

Figure 3. The contour for the Nyquist criterion with HTFs in an $s$ plane that is denoted by $\bar{N}_{f}$. The notation " $\times$ " corresponds to the poles of $\mathcal{H}(s)$.

$-1 / k$ is encircled in counterclockwise direction $n_{p}$ times, which is equal to the number of right half-plane poles of truncated HTFs in the fundamental strip.

Poles of LTP systems in an s plane. The poles of the LTP system can be found by checking the points where the HTFs are not analytical. As can be seen from (19), poles of an LTP system can be described based on the poles of its corresponding LTI transfer functions, $H_{k}(s)$. Hence, in a complex $s$ plane, the solution of the following eigenvalue problem yields the poles of the LTP system:

$$
\{s \mathcal{I}-(\mathcal{A}-\mathcal{N})\} \mathcal{V}=0
$$

The solution to above equation gives an infinite number of poles. To obtain the right half-plane poles of the LTP systems considered in Theorem 1, we look at the poles that are placed in the fundamental strip of HTFs. Thus, we obtain the Nyquist diagram of known LTP systems by computing eigenvalues of analytically derived HTFs after truncation and plotting them on the complex plane.

\section{Stability analysis of unknown LTP systems via estimated HTFs}

In this section, we explain how we can utilize these datadriven techniques to generate the Nyquist diagram for the unknown LTP system. To this end, we first utilize the datadriven techniques explained in previous sections to estimate the HTFs of the open-loop LTP system. Then, we compute the eigenlocus of estimated HTFs by using standard numerical methods. One key difference here is the structure of the HTFs matrices between the analytically derived and estimated ones. Estimated HTFs are actually a subset of the analytically derived HTFs, which were used for obtaining the Nyquist diagrams.

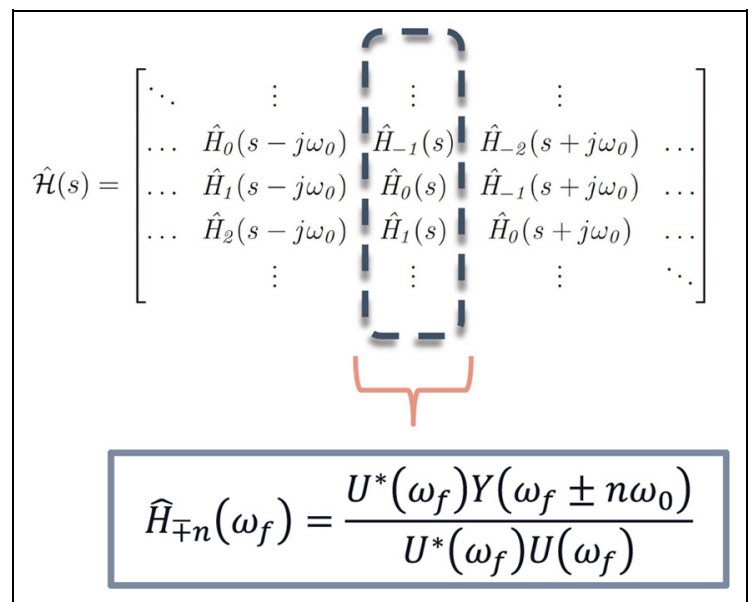

Figure 4. The relation between estimated HTFs and required HTF to plot the Nyquist diagram.

The relation between estimated HTFs $\left(\hat{H}_{\mp n}\left(\omega_{f}\right)\right)$ with the data-driven approach and the required HTF structure to obtain Nyquist diagram is shown in Figure 4. The Nyquist diagram is obtained via the eigenlocus of the doubly infinite matrix structure, $\hat{\mathcal{H}}(s)$, illustrated in Figure 4. To obtain the eigenlocus of $\hat{\mathcal{H}}(s)$, first we truncate it to the order of $N$ and compute the eigenvalues of these matrices for $s=j \omega$ and $\omega$ varying through $-\omega_{0} / 2<\omega<\omega_{0} / 2$. However, our estimated HTFs with data-driven approach, $\left(\hat{H}_{\mp n}\left(\omega_{f}\right)\right)$, will preserve the structure illustrated in the dashed rectangle in Figure 4.

Here, our aim is to obtain the structure of $\hat{\mathcal{H}}(s)$ with estimated HTFs, $\left(\hat{H}_{\overline{+} n}\left(\omega_{f}\right)\right)$. If we look at the structure of HTF matrix shown in Figure 4, which forms the basis of Nyquist diagram, $\hat{\mathcal{H}}(s)$, includes modulated LTI transfer functions from $\hat{H}_{ \pm n}\left(-\omega_{0} / 2-N \omega_{0}\right)$ to the $\hat{H}_{ \pm n}\left(\omega_{0} / 2+N \omega_{0}\right)$. On the other hand, when we perform system identification for $s=j \omega$ and $\omega$ varying through $-\omega_{0} / 2<\omega<\omega_{0} / 2$, estimated HTFs consist of LTI transfer functions from $\hat{H}_{ \pm n}\left(-\omega_{0} / 2\right)$ to the $\hat{H}_{ \pm n}\left(\omega_{0} / 2\right)$. Therefore, to make estimated HTFs suitable for plotting a Nyquist diagram as it will have same structure as $\hat{\mathcal{H}}(s)$, we perform system identification methods for $s=j \omega$ and $\omega$ varying through $\left(\frac{-\omega_{0}}{2}-N \omega_{0}\right)$ to $\left(\frac{\omega_{0}}{2}+N \omega_{0}\right)$. Here, to obtain an appropriate value for the truncation number of $N$, we increase it until the result of estimated HTFs does not change, and the additional eigenvalues ended up in the origin (Möllerstedt, 2000). Then, by placing estimated HTFs in this frequency range in their corresponding locations in the HTF structure, we can successfully plot the Nyquist diagram of unknown LTP systems with estimated HTFs by using only input and output data.

\section{Algorithm for controller design based on HTFs}

In this section, our goal is to analyze the stability of LTP systems by closing the feedback loop with P-, PD- and PID-type controllers by using the Nyquist stability criterion. One key problem we will be focusing on in this section is to formulate the stability problem as a function of a feedback gain $K_{p}$ and determine the range of $K_{p}$ that stabilizes the closed-loop 
system with a single Nyquist stability test. We note that the methodology presented here could easily be extended to other types of controllers, e.g. lead/lag controllers, etc.

HTF representation of controllers. Despite the fact that we are dealing with time-invariant P, PD and PID controllers, we need to have their HTF-like representations for the sake of our HTF-based stability analysis. To accomplish this, we utilize the same approach used for lifting the time-periodic system matrices to obtain Toeplitz form structures of the controllers. However, one needs to keep in mind that the lifted controllers do not have any spectral interactions and their HTFs representations can be obtained as follows (Bellinaso et al., 2011; Scapini et al., 2012):

$$
\mathcal{H}_{C}(s)=\left[\begin{array}{ccccc}
\ddots & \vdots & \vdots & \vdots & \\
\ldots & C\left(s-j \omega_{0}\right) & 0 & 0 & \ldots \\
\cdots & 0 & C(s) & 0 & \ldots \\
\cdots & 0 & 0 & C\left(s+j \omega_{0}\right) & \ldots \\
& \vdots & \vdots & \vdots & \ddots
\end{array}\right]
$$

where $C(s)$ is the transfer function of the LTI form of the controller. To apply Nyquist stability analysis, we have to examine the eigenvalues of $\mathcal{H}(s)=\mathcal{H}_{C}(s) \mathcal{H}_{P}(s)$.

PD-type controller. The main objective of this section is to illustrate adoption of the structure of a PD controller into the closed-loop LTP feedback system, which is shown in Figure 5. The PD controller structure is given as

$$
C_{p d}(s)=K_{p}+K_{d} s=K_{p} \underbrace{(1+\alpha s)}_{C(s)}
$$

where $\alpha=K_{d} / K_{p}$. By using LTI $C(s)$, the HTF form of the controller is obtained as illustrated in (27). Here, the reason behind extracting a $K_{p}$ parameter from the HTF form of the controller is to design the $K_{p}$ parameter as feedback gain as is shown in Figure 5 and, thus, for different values of $\alpha$, by applying the Nyquist stability test to compute the range of $K_{p}$, which stabilizes the closed-loop system. The important point is that by designing the $K_{p}$ parameter of the PD controller as a feedback gain and applying the Nyquist stability test provides stability analysis along the line $\alpha=K_{d} / K_{p}$ with one test instead of requiring a stability analysis for each single point in the $\left(K_{p}, K_{d}\right)$ plane. Hence, a two-dimensional stability

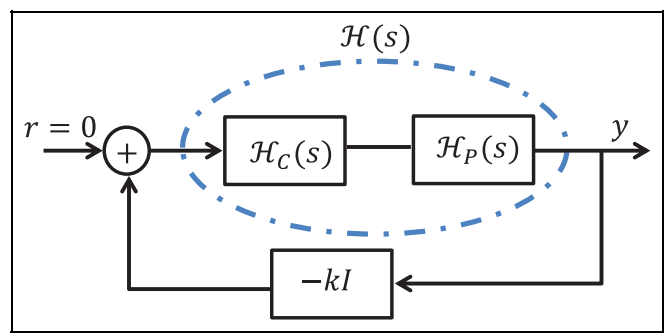

Figure 5. Closed-loop feedback system with controller. problem that depends on the points in the $\left(K_{p}, K_{d}\right)$ plane can be reduced successfully to the one-dimensional stability analysis problem investigating stability along the line of $\alpha=K_{d} / K_{p}$ (see Remark 1).

Remark 1. Note that the structure of the PID controller is given in the following:

$$
C_{p i d}(s)=K_{p}+\frac{K_{i}}{s}+K_{d} s=K_{p} \underbrace{\left(1+\frac{\alpha_{1}}{s}+\alpha_{2} s\right)}_{C(s)}
$$

where $\alpha_{1}=K_{i} / K_{p}$ and $\alpha_{2}=K_{d} / K_{p}$. Similarly, designing the $K_{p}$ parameter as a feedback gain, the Nyquist stability test can be applied for different values of $\left(\alpha_{1}, \alpha_{2}\right)$ and the range found for $K_{p}, K_{i}$ and $K_{d}$ parameters, which stabilize the LTP system. Here, the three-dimensional stability analysis problem can be reduced successfully to the two-dimensional problem.

\section{Examples and simulation results}

\section{Stability analysis and control of known unstable LTP system via theoretical HTFs}

In this section, our goal is to investigate the stability analysis of known LTP systems and design P, PD and PID controllers accordingly by using the Nyquist stability criterion. According to the stability analysis results of LTP systems including the controller, we will obtain the GM and PM of the closed-loop system from the Nyquist diagram with respect to the specific parameters of controllers. As a result of these measurements, we choose some controllers that enhance the performance of the system and we perform various simulation studies with chosen controllers to show the performance of the controllers in the time domain.

To implement these procedures, we use a well-known lossy Mathieu example whose system matrices are given in the following:

$$
\begin{aligned}
& A(t)=\left[\begin{array}{cc}
0 & 1 \\
-\left(5-\beta \cos \omega_{0} t\right) & -2 \zeta
\end{array}\right] \\
& B=\left[\begin{array}{l}
0 \\
1
\end{array}\right], \quad C=\left[\begin{array}{ll}
1 & 0
\end{array}\right]
\end{aligned}
$$

The parameters of this system are chosen as $\omega_{0}=2, \beta=8$ and $\zeta=0.2$. The poles of the open-loop system in $s$ plane are obtained as $s_{1}=0.3931$ and $s_{2}=-0.7931$ by computing eigenvalues of $\mathcal{A}-\mathcal{N}$ in the fundamental strip. This system has one pole at the right half-plane.

P-type controller. The lossy Mathieu equation in (30) is unstable with the parameters given above because it includes one pole at right half-plane. To make the system stable, the P-type controller can be designed by using the Nyquist criterion. According to Theorem 1, because the system has one pole at the right half-plane, the closed-loop system will be stable if and only if the $-1 / k$ point is encircled only once in counterclockwise direction. To calculate $K_{p}$ values of the $\mathrm{P}$ controller that stabilizes the system, HTF of the LTP system, 


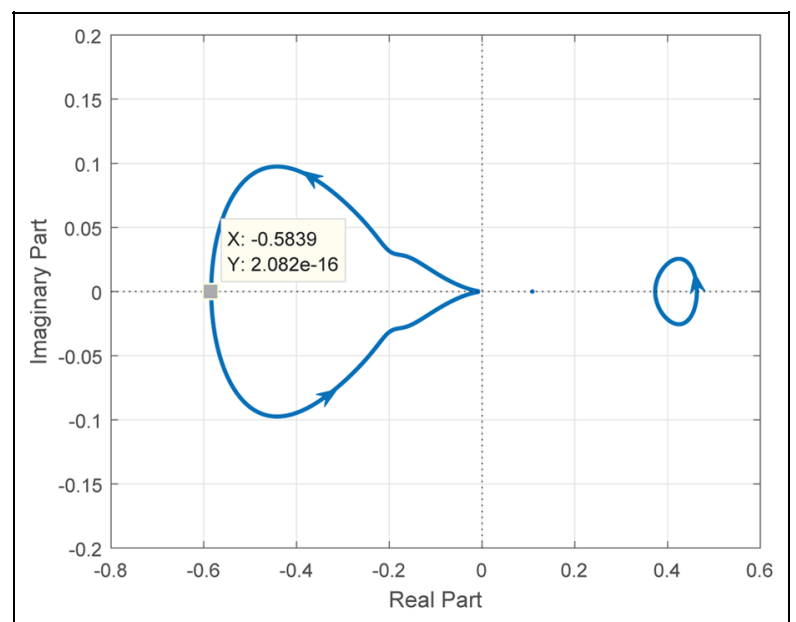

Figure 6. Nyquist diagram of an open-loop HTF, $\mathcal{H}_{p}$.

$\mathcal{H}_{p}$ is computed as in Equation (18) and the Nyquist diagram of $\mathcal{H}_{p}$ is obtained as in Figure 6. In the Nyquist diagram of the lossy Mathieu equation shown in Figure 6, the negative real axis is encircled counterclockwise once for the values of $-1 / k \in[-0.58,0]$. Hence, the range of $K_{p}$ values that stabilize the closed-loop system is computed as $[1.71, \infty)$.

According to the values of $K_{p}$ that stabilizes the system, GM and PM graphics of the closed-loop system are obtained in Figure 7 (A) and (B), respectively, from the Nyquist diagram by using classical methods. If we investigate GM and PM of the closed-loop system with respect to $K_{p}$, as GM changes between $(0.01,1], \mathrm{PM}$ varies between $\left(2^{\circ}, 13^{\circ}\right.$. However, the PM of the closed-loop system does not vary much and as can be seen from Figure 7 (B) the PM is not sufficiently high. Therefore, to enhance the performance and stabilize the system, alternative controllers can be designed and the performances of these controllers on the system can be investigated.

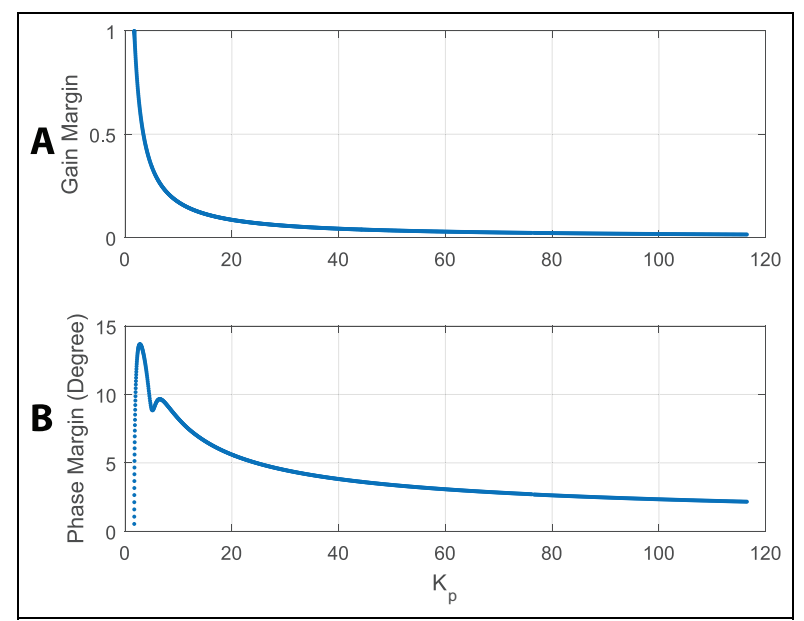

Figure 7. (A) GM and (B) PM graphs with respect to the $K_{p}$ values that stabilize the system.

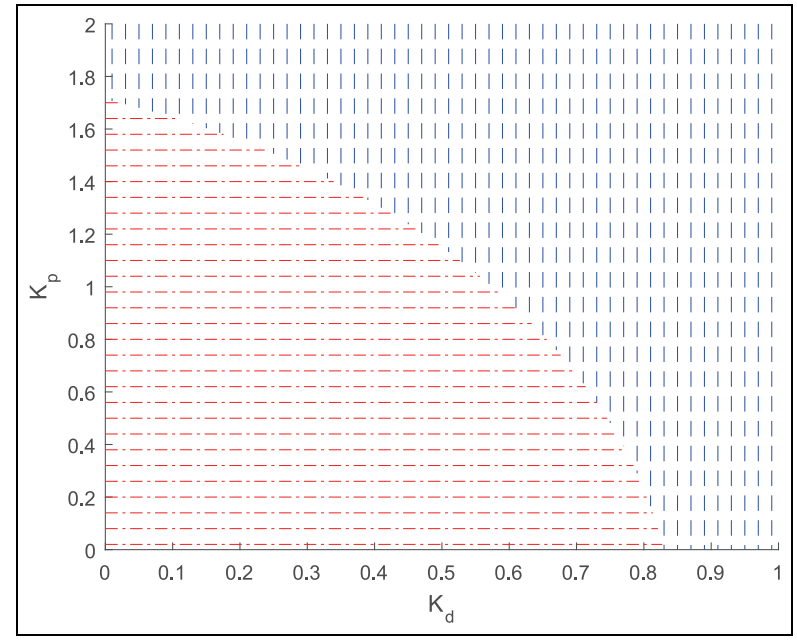

Figure 8. Stable and unstable regions of the closed-loop system with respect to the value of $K_{p}$ and $K_{d}$. Red (horizontally dashed) lines illustrate unstable regions and blue (vertically dashed) lines include stable regions of the closed-loop system.

PD-type controller. The main objective of this section is to design a PD controller that stabilizes the unstable LTP system and enhances the performance of the system in terms of PM and GM. In this respect, we first analyze the stability of LTP unstable Mathieu example given in the previous section that includes the PD controller with respect to the $\left(K_{p}, K_{d}\right)$ controller parameters via the Nyquist stability criterion. We then obtain GM and PM graphs of the closed-loop system according to the $\left(K_{p}, K_{d}\right)$ parameters that stabilize the unstable system by using the Nyquist diagram. For different values of $\alpha=K_{d} / K_{p}$ between $[0.01,100]$ changing with 0.01 steps, we apply the Nyquist stability test and we obtain the range of $K_{p}$ that stabilizes the closed-loop system. By using $K_{d}=\alpha K_{p}$, we also calculate the range of $K_{d}$. According to this, we obtain Figure 8 , which includes stable and unstable regions of the closed-loop system with respect to the $\left(K_{p}, K_{d}\right)$ parameters of the PD controller via the Nyquist stability criterion.

In Figure 8, blue (vertically dashed) lines illustrate the region where the closed-loop system is stable for corresponding $\left(K_{p}, K_{d}\right)$ values and red (horizontally dashed) lines include the controller parameters which leave the system as unstable. A significant point is that the closed-loop stability analysis of a LTP system with a controller is achieved along the line $\alpha=K_{d} / K_{p}$, where $\alpha \in[0.01,100]$ by designing he $K_{p}$ parameter as a feedback gain instead of checking the stability for a single point in the $\left(K_{p}, K_{d}\right)$ plane. After finding the range of $\left(K_{p}, K_{d}\right)$, which makes the closed-loop system stable, for the purpose of investigating the performance of the system, we compute the GM and PM of the system with a controller by using the Nyquist diagram. Thus, we aim to test a PD controller having $\left(K_{p}, K_{d}\right)$ parameters that may provide a possible performance improvement for the system given by the damped Mathieu equation. In this respect, the threedimensional graphs of GM and PM of the closed-loop system with respect to the value of $\left(K_{p}, K_{d}\right)$ parameters that stabilize the system are given in Figures 9 and 10, respectively. In these 


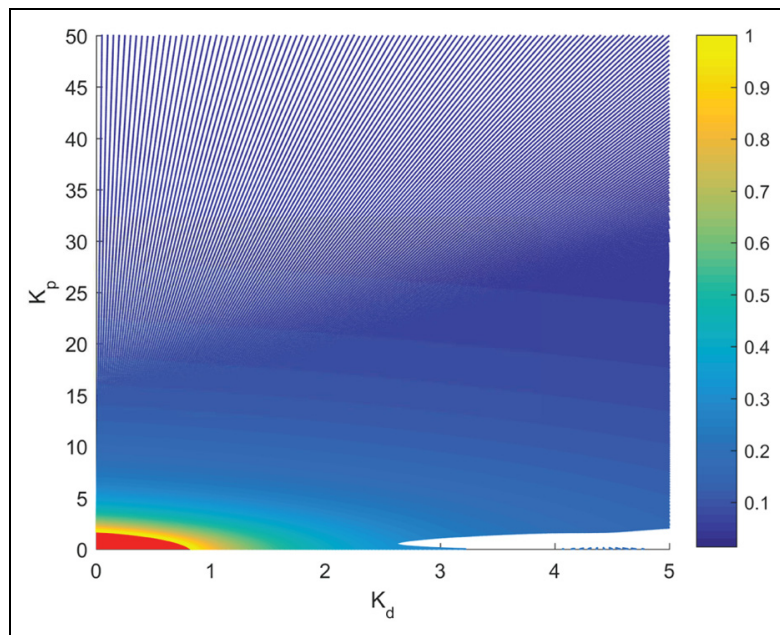

Figure 9. GM of the closed-loop system with respect to $K_{p}$ and $K_{d}$ values. The red region illustrates the unstable regions.

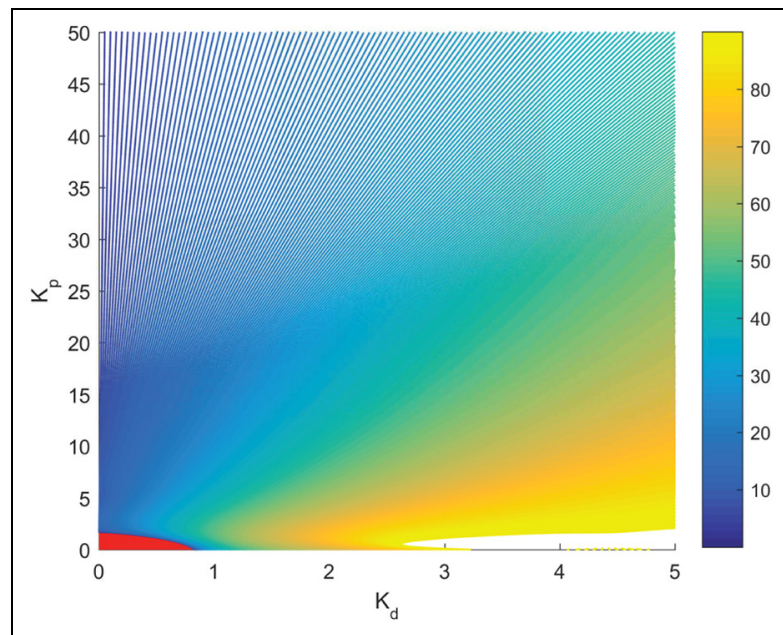

Figure 10. PM of the closed-loop system with respect to $K_{p}$ and $K_{d}$ values. The red region illustrates the unstable regions.

figures, the $x$-axis corresponds to $K_{p}$ values and the $y$-axis belongs to $K_{d}$ values of the PD controller. The GM and PM values are shown via a colour map. The red regions illustrated in Figures 9 and 10 belong to the unstable regions, which are also shown in Figure 8.

If we examine Figure 9, the GM has high values in the locations where $K_{p}$ and $K_{d}$ are close to the origin. However, PM increases in the regions where $K_{d}$ also increases. According to this, it can be concluded that there is a trade-off between GM and PM.

When GM and PM are taken into consideration, as GM and PM will be between $[0.25,0.6]$ and $\left[40^{\circ}, 90^{\circ}\right]$, respectively, we design two different PD controllers that yield PM and GM close to the boundaries of these ranges. The first controller is given as $C_{P D_{1}}=2.3+0.75 \mathrm{~s}$ where the resulting $\mathrm{GM}$ and $\mathrm{PM}$ are found as 0.55 and $43^{\circ}$, respectively. The second controller
Table 2. Performance of $C_{P D_{1}}$ and $C_{P D_{2}}$ in time domain simulations

\begin{tabular}{lcccll}
\hline Controller (PD) & $\mathrm{GM}$ & $\mathrm{PM}\left({ }^{\circ}\right)$ & $P O(\%)$ & $T_{r}(\mathrm{sec})$ & $T_{s}(\mathrm{sec})$ \\
\hline $2.3+0.75 \mathrm{~s}$ & 0.55 & 43 & 29 & 1.5 & 9.33 \\
$1.6+3 \mathrm{~s}$ & 0.25 & 87 & 2 & 1.7 & 2.3 \\
\hline
\end{tabular}

is given as $C_{P_{2}}=1.6+3 s$ where the resulting GM and PM are found as 0.27 and $87^{\circ}$, respectively.

Time domain simulations with $C_{P D_{1}}$ and $C_{P D_{2}}$. In this section, we evaluate the performance of the PD controllers that are designed above in terms of percentage overshoot $(P O)$, rising time $\left(T_{r}\right)$ and settling time $\left(T_{s}\right)$ criterion. In these simulations, the input is equal to zero and corresponding output is the first state variable, $x$. We observe that when $K_{d}$ increases, PM increases and time domain simulations show that the percentage overshoot and settling time also decrease, as shown in Table 2.

\section{Controller design of unknown LTP system via estimated HTFs with data-driven approach}

In this section, we illustrate the design of a controller to enhance the performance of an unknown Mathieu example by using the Nyquist diagram of estimated HTFs, which is obtained with sum-of-cosine input signal and its corresponding data. The system matrices of a stable lossy Mathieu equation are given by (30) with $\alpha=4, \omega_{0}=2 \pi, \zeta=0.2$. The poles of the open-loop system in $s$ plane are obtained as $s_{1}=-0.2-2.2271 i$ and $s_{2}=-0.2+2.2271 i$ by computing eigenvalues of $\mathcal{A}-\mathcal{N}$ in fundamental strip. Note that this system is stable as it has no pole at the right half-plane.

As this system is stable, we can estimate the HTF by using data-driven system identification methods. For this example, we first estimate the HTFs of the system. Here, as an input, we apply a sum-of-cosine input signal that includes different frequency cosine signals to the Matlab Simulink model of the Mathieu example and we compute the corresponding output. We estimate HTFs from these inputs-outputs by using the data-driven system identification procedure detailed in the previous sections. Then, we modify it to obtain the HTF structure illustrated in Figure 4, which is required to obtain the Nyquist diagram. The important point here is that we do not have any information about the state space model of the example, we only have the exciting input signal and their corresponding outputs. Here, the Nyquist diagram of the unknown Mathieu example that is obtained from the eigenlocus of estimated HTFs by using input output data is shown in Figure 11.

When we investigate the Nyquist diagram of the unknown Mathieu example, there are two real-axis crossing points at -0.3062 and -0.1569 . According to Theorem 1 , to satisfy stability, the $-1 / k$ point should not be encircled. Regarding this, stabilizing feedback gain values are obtained as follows:

$$
0<k<3.33 \text { and } 6.37<k<\infty
$$




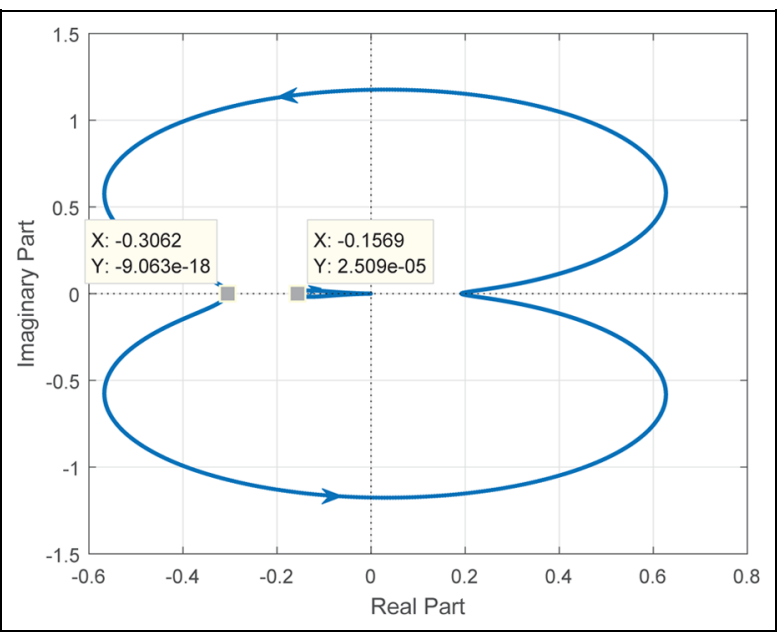

Figure I I. Nyquist diagram of the stable Mathieu equation that is obtained from the eigenlocus of estimated HTFs.

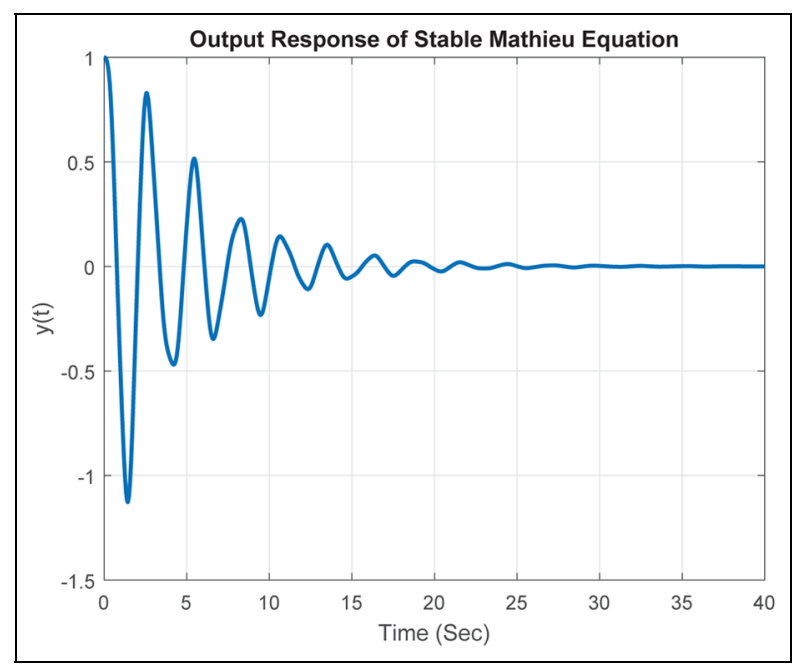

Figure 12. Output response of the open-loop stable Mathieu equation in the time domain with input zero.

The open-loop output response of the stable Mathieu equation is illustrated in Figure 12.

If we investigate the time domain performance of the open-loop stable Mathieu equation, whereas percentage overshoot is obtained as $P O=108 \%$ and settling time is acquired as $T_{s}=24 \mathrm{~s}$. To enhance the performance of this system, we also design PD controllers by using the procedure explained in (28) by using the corresponding Nyquist diagram. To investigate the robustness and performance of the system, we obtain the GM and PM of the closed-loop system via the Nyquist diagram that is plotted according to the estimated HTF with respect to parameters of the PD controller. The three-dimensional graphs of GM and PM of the closed-loop system with respect to the value of $\left(K_{p}, K_{d}\right)$ parameters are illustrated in Figures 13 and 14, respectively.

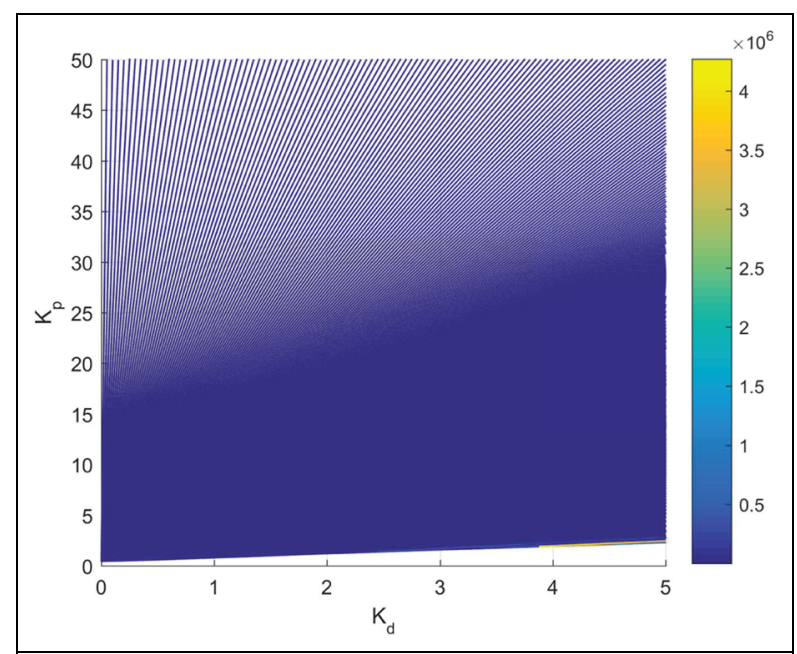

Figure 13. GM of the closed-loop stable Mathieu system with respect to $K_{p}$ and $K_{d}$ values.

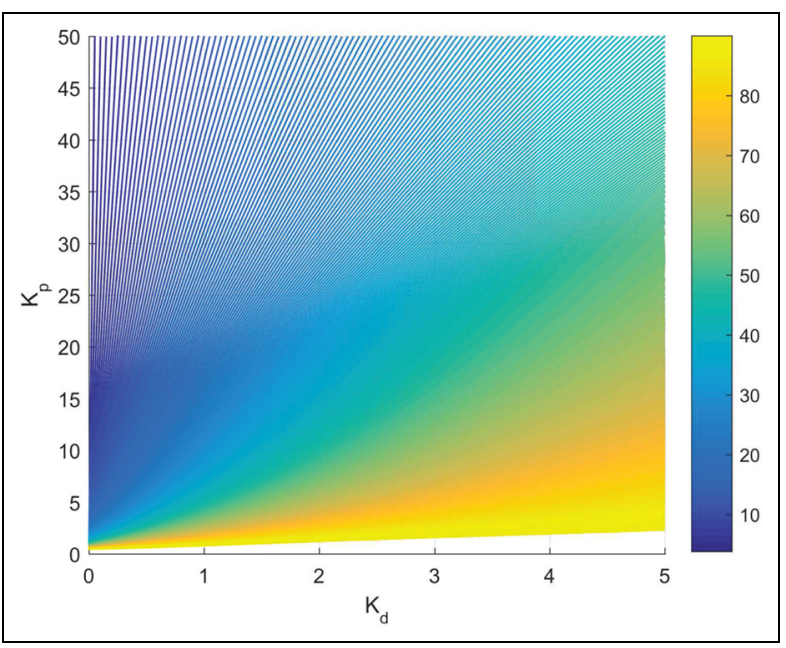

Figure 14. PM of the closed-loop stable Mathieu system with respect to $K_{p}$ and $K_{d}$ values.

According to the obtained results, the GM and PM are found in the ranges $[0.6, \gg 1]$ and $\left[40^{\circ}, 90^{\circ}\right]$, respectively. Then, we design two different PD controllers that yield PM and GM close to the boundaries of these ranges. Accordingly, the first controller is given as $C_{P D_{1}}=5+3 s$ where the resulting GM and PM are found as 80 and $75^{\circ}$, respectively. The second controller is given as $C_{P D_{2}}=2.4+5 \mathrm{~s}$ where the resulting GM and PM are found as $>10^{6}$ and $89^{\circ}$, respectively.

Here, we evaluate the performance of these PD controllers which are designed above in terms of percentage overshoot $(P O)$, rising time $\left(T_{r}\right)$ and settling time $\left(T_{s}\right)$ criterion. Time domain simulation results of $C_{P D_{1}}$ and $C_{P D_{2}}$ which are designed regarding GM and $\mathrm{PM}$ graphs are illustrated in Figure 15 -A and Figure 15 -B respectively. Corresponding results are also given in Table 3. If we compare the closed 


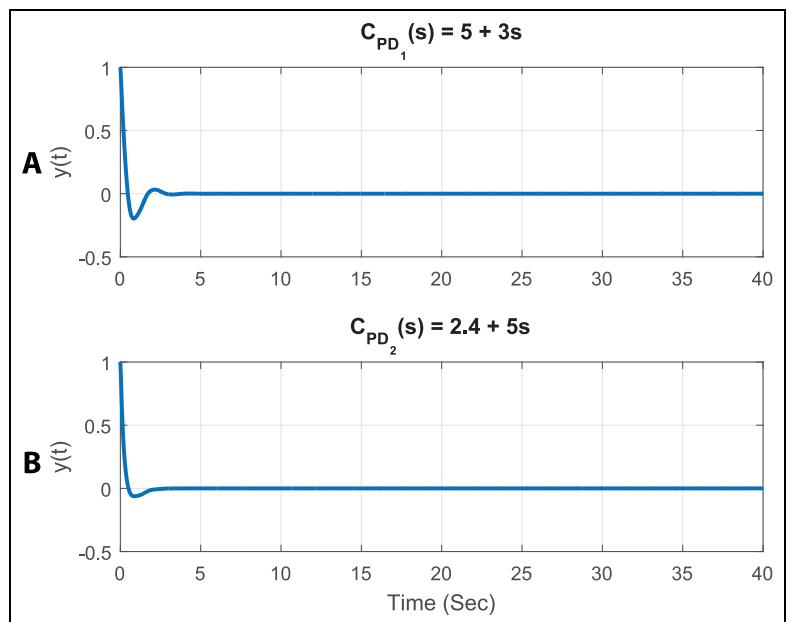

Figure 15. Output response of $C_{P D_{1}}$ and $C_{P D_{2}}$ in time domain simulations.

Table 3. The performance of $C_{P D_{1}}$ and $C_{P D_{2}}$ in time domain simulations.

\begin{tabular}{llllll}
\hline Controller (PD) & GM & PM $\left(^{\circ}\right)$ & $P O(\%)$ & $T_{r}(\mathrm{sec})$ & $T_{s}(\mathrm{sec})$ \\
\hline $5+3 s$ & $\ggg 1$ & 75 & 19 & 0.8 & 3.5 \\
$2.4+5 s$ & $\ggg 1$ & 89 & 5 & 0.9 & 1.9 \\
\hline
\end{tabular}

loop time domain simulation results of stable Mathieu example including PD controller with time domain simulation of open loop stable Mathieu example without controller, we can conclude that, stability robustness and performance of the system are enhanced regarding percentage overshoot and settling time criterion. As a result, by using only input and output data of unknown Mathieu example, we estimate the HTF. Then, by applying Nyquist test via estimated HTF, we design two different PD controllers which can achieve successful results in enhancing the robustness and improving the performance of stable system.

\section{Conclusion}

In this paper, we have investigated the problem of stabilization and control of LTP systems. We utilized HTFs and the Nyquist stability criterion as basic tools in our analysis. We first considered the stabilization of a known LTP system by using these tools. Then we considered an unknown but stable LTP system from an input/output point of view. We proposed a method to estimate the HTFs of such a system. Then based on these estimations, we have proposed a novel controller design method to improve some performance measures of associated closed-loop system.

The main contributions of this work are as follows. First of all, we investigated the stability of known LTP systems by utilizing the Nyquist stability criterion that is based on HTFs and we designed P, PD and PID controllers that stabilize and enhance the performance of unstable LTP systems by using
Nyquist diagrams. While designing PD or PID controllers, we extract the $K_{p}$ parameter from the HTF form of controller and design this parameter as feedback gain. The important point here is that designing the $K_{p}$ parameter of the PD controller as a feedback gain allow us to apply the Nyquist stability test along the line $\alpha=K_{d} / K_{p}$. By doing so, a single test for each $\alpha$ value provides stability analysis along the associated $K_{d} / K_{p}$ line. Hence, two-dimensional stability problem that depends on the points in the $\left(K_{p}, K_{d}\right)$ plane can be reduced successfully to the one-dimensional stability analysis problem as investigating stability along the line of $\alpha=K_{d} / K_{p}$. Hence, we analyzed the stability of an known LTP system and design P, PD and PID controllers to provide stability and increase the performance of the system.

The second contribution of this work is related to the control of unknown LTP systems. For the unknown LTP systems whose state space model may not be available, we seek to design a controller by using similar methodology for the known systems. To achieve that, we first estimated the HTFs of unknown LTP systems by using a data-driven system identification procedure. In this regard, we designed a sum-ofcosine input signal including different frequencies whose corresponding output components do not coincide. By using input-output relation, we obtained the estimated HTFs of the unknown system. Then, we designed P and PD controllers to enhance the performance and increase the robustness by using Nyquist diagram of estimated HTFs. As a final work, we illustrated the performances of designed controllers in time domain simulations for both known and unknown LTP systems.

\section{Declaration of Conflicting Interests}

The author(s) declared no potential conflicts of interest with respect to the research, authorship, and/or publication of this article.

\section{Funding}

The author(s) disclosed receipt of the following financial support for the research, authorship, and/or publication of this article: This work was supported by funding from The Scientific and Technological Research Council of Turkey (TÜBITAK) granted to Elvan Kuzucu.

\section{ORCID iD}

Ismail Uyanik (iD https://orcid.org/0000-0002-3535-5616

\section{References}

Allen MS, Sracic MW, Chauhan S and Hansen MH (2011) Outputonly modal analysis of linear time-periodic systems with application to wind turbine simulation data. Mechanical Systems and Signal Processing 25(4): 1174-1191.

Anderson BD and Moore JB (2007) Optimal Control: Linear Quadratic Methods. Englewood Cliffs, NJ: Prentice-Hall.

Arcara P, Bittanti S and Lovera M (1997) Active control of vibrations in helicopters by periodic optimal control. Proceedings of the 1997 IEEE International Conference on Control Applications. Piscataway, NJ: IEEE, pp. 730-735. 
Athans M (1971) The role and use of the stochastic linear-quadraticGaussian problem in control system design. IEEE Transactions on Automatic Control 16(6): 529-552.

Barmanj JF and Katzenelson J (1974) A generalized Nyquist-type stability criterion for multivariable feedback systems. International Journal of Control 20(4): 593-622.

Bellinaso LV, Scapini RZ and Michels L (2011) Modeling and analysis of single phase full-bridge PFC boost rectifier using the LTP approach. In: Brazilian Power Electronics Conference, pp. 93-100.

Bittanti S and Colaneri P (2000) Invariant representations of discretetime periodic systems. Automatica 36(12): 1777-1793.

Bittanti S, Fronza G and Guardabassi G (1973) Periodic control: A frequency domain approach. IEEE Transactions on Automatic Control 18(1): 33-38.

Bottasso C and Cacciola S (2015) Model-independent periodic stability analysis of wind turbines. Wind Energy 18(5): 865-887.

DaCunha JJ and Davis JM (2011) A unified Floquet theory for discrete, continuous, and hybrid periodic linear systems. Journal of Differential Equations 251(11): 2987-3027.

Desoer C and Wang YT (1980) On the generalized Nyquist stability criterion. IEEE Transactions on Automatic Control 25(2): 187-196.

Farkas M (2013) Periodic motions, vol. 104. Springer Science \& Business Media.

Goos J and Pintelon R (2016) Continuous-time identification of periodically parameter-varying state space models. Automatica 71: 254-263.

Hall SR and Wereley NM (1990) Generalized nyquist stability criterion for linear time periodic systems. In: American Control Conference, pp. 1518-1525.

Hidır EK (2017) Identification, stability analysis and control of linear time periodic systems via harmonic transfer functions. Master's Thesis, Bilkent University.

Hwang S (1997) Frequency Domain System Identification of Helicopter Rotor Dynamics Incorporating Models With Time Periodic Coefficients. PhD Thesis, University of Maryland.

Jakobsen C, Camino J and Santos F (2013) Rotor-blade vibration control using a periodic LQR controller. Proceeding Series of the Brazilian Society of Computational and Applied Mathematics, Vol. $1(1)$.

Kwon J, Wang X, Blaabjerg F and Bak CL (2017) Frequency-domain modeling and simulation of dc power electronic systems using harmonic state space method. IEEE Transactions on Power Electronics 32(2): 1044-1055.

Logan D, Kiemel T and Jeka JJ (2016) Using a system identification approach to investigate subtask control during human locomotion. Frontiers in Computational Neuroscience 10: 146.

MacFarlane AG and Postlethwaite I (1977) The generalized Nyquist stability criterion and multivariable root loci. International Journal of Control 25(1): 81-127.

Mckillip R Jr (1985) Periodic control of the individual blade control helicopter rotor. Vertica 9: 199-225.
Möllerstedt E (2000) Dynamic analysis of harmonics in electrical systems. PhD Thesis, Lund Institute of Technology.

Mollerstedt E and Bernhardsson B (2000a) A harmonic transfer function model for a diode converter train. In: IEEE Power Engineering Society Winter Meeting, vol. 2. IEEE, pp. 957-962.

Mollerstedt E and Bernhardsson B (2000b) Out of control because of harmonics - an analysis of the harmonic response of an inverter locomotive. IEEE Control Systems 20(4): 70-81.

Owens D, Li LM and Banks SP (2004) Multi-periodic repetitive control system: A Lyapunov stability analysis for MIMO systems. International Journal of Control 77(5): 504-515.

Richards JA (1983) Analysis of periodically time-varying systems. Berlin: Springer-Verlag.

Sandberg H, Mollerstedt E, et al. (2005) Frequency-domain analysis of linear time-periodic systems. IEEE Transactions on Automatic Control 50(12): 1971-1983.

Scapini RZ, Bellinaso LV and Michels L (2012) Stability analysis of half-bridge rectifier employing LTP approach. In: IECON 201238th Annual Conference on IEEE Industrial Electronics Society, pp. 780-785.

Siddiqi A (2001) Identification of the harmonic transfer functions of a helicopter rotor. Master's Thesis, Massachusetts Institute of Technology.

Sracic MW and Allen MS (2011) Method for identifying models of nonlinear systems using linear time periodic approximations. Mechanical Systems and Signal Processing 25(7): 2705-2721.

Uyanik I (2017) Identification of legged locomotion via model-based and data-driven approaches. $\mathrm{PhD}$ Thesis, Bilkent University.

Uyanik I, Ankarali MM, Cowan NJ, Morgül Ö and Saranli U (2015) Toward data-driven models of legged locomotion using harmonic transfer functions. In: Proceedings of the IEEE International Conference on Advanced Robotics, pp. 357-362.

Uyanik I, Ankarali MM, Cowan NJ, Saranli U and Morgül Ö (2016a) Identification of a vertical hopping robot model via harmonic transfer functions. Transactions of the Institute of Measurement and Control 38(5): 501-511.

Uyanik I, Saranli U, Ankarali MM and Morgül Ö (2017) Frequency domain subspace identification of linear time periodic (LTP) systems. IEEE Transactions on Automatic Control, in press.

Uyanik I, Saranli U, Morgül Ö and Ankarali MM (2016b) Parametric identification of hybrid linear-time-periodic systems. IFAC-PapersOnLine 49(9): 7-12.

Verhaegen M and Yu X (1995) A class of subspace model identification algorithms to identify periodically and arbitrarily timevarying systems. Automatica 31(2): 201-216.

Wereley NM (1990) Analysis and control of linear periodically time varying systems. $\mathrm{PhD}$ Thesis, Massachusetts Institute of Technology.

Wereley NM and Hall SR (1990) Frequency response of linear time periodic systems. In: Proceedings of the IEEE International Conference on Decision and Control, pp. 3650-3655. 